\title{
Impossibility to produce arbitrary non-Gaussian states using zero-mean Gaussian states and partial photon number resolving detection
}

\author{
Christos N. Gagatsos $\odot^{1}$ and Saikat Guha ${ }^{1,2}$ \\ ${ }^{1}$ James C. Wyant College of Optical Sciences, University of Arizona, Tucson, Arizona 85721, USA \\ ${ }^{2}$ Department of Electrical and Computer Engineering, University of Arizona, Tucson, Arizona 85721, USA
}

(Received 17 May 2020; revised 15 November 2021; accepted 23 November 2021; published 15 December 2021)

\begin{abstract}
Gaussian states and measurements collectively are not powerful-enough resources for quantum computing, as any Gaussian dynamics can be simulated efficiently, classically. However, it is known that any one nonGaussian resource-a state, a unitary operation, or a measurement-together with Gaussian unitaries, makes for universal quantum resources. Photon number resolving (PNR) detection, a readily realizable non-Gaussian measurement, has been a popular tool to try and engineer non-Gaussian states for universal quantum processing. In this paper, we consider PNR detection of a subset of the modes of a zero-mean pure multimode Gaussian state as a means to herald a target non-Gaussian state on the undetected modes. This is motivated from the ease of scalable preparation of Gaussian states that have zero mean, using squeezed vacuum and passive linear optics. We calculate upper bounds on the fidelity between the actual heralded state and the target state. We find that this fidelity upper bound is $1 / 2$ when the target state is a multimode coherent cat-basis cluster state, a resource sufficient for universal quantum computing. This proves that there exist non-Gaussian states that are not producible by this method. Our fidelity upper bound is a simple expression that depends only on the target state represented in the photon-number basis, which could be applied to other non-Gaussian states of interest.
\end{abstract}

DOI: 10.1103/PhysRevResearch.3.043182

\section{INTRODUCTION}

Production of non-Gaussian quantum states of light, and all-optical realization of non-Gaussian quantum unitary operations, are critical for most applications of photonic quantum information processing, e.g., universal photonic quantum computation [1], quantum-enhanced receivers for optical communications [2,3], all-optical quantum repeaters for long-distance entanglement distribution [4-6], and quantumenhanced optical sensing [7-12].

Gaussian states and Gaussian unitaries, produced by the action of linear and quadratic Hamiltonians on the vacuum state, have efficient and complete mathematical representations [13-15]. Non-Gaussian states is a vast set-it consists of states generated via the action, on the multimode vacuum state, of a unitary with Hamiltonian that is a thirdor higher-order polynomial in the field operators. Therefore, non-Gaussian states are inherently underexplored and their general representations less understood.

Deterministic realization of non-Gaussian unitary operations, such as the self-Kerr gate $[16,17]$ and the cubic-phase gate [18], is near impossible at optical frequencies [19]. The extreme resource inefficiency resulting from this deficiency, combined with the fact that Gaussian states and Gaussian

Published by the American Physical Society under the terms of the Creative Commons Attribution 4.0 International license. Further distribution of this work must maintain attribution to the author(s) and the published article's title, journal citation, and DOI. unitaries are a classically simulable resource [20], have kept all-photonic quantum computing from being pursued as one of the top contenders for quantum computing, for decades since their invention, despite their obvious importance in optical communications and sensing applications, and not requiring quantum transduction for networking far-flung quantum processors-a major benefit unique to photonic quantum encodings.

Recent advances in discrete variable (DV), i.e., singlephoton-qubit based, quantum computing [16] have revealed that deterministic production of even small non-Gaussian resource states (e.g., a three-photon-entangled GHZ state) can enable resource-efficient universal photonic quantum computing, despite two-qubit gates being inherently probabilistic $[1,21]$. However, a systematic understanding of efficient production of even such simple non-Gaussian states as GHZ states and realization of simple two-qubit non-Gaussian measurements (e.g., Bell-state measurements) required for DV quantum computing has proven extremely difficult [22].

A major attraction of continuous variable (CV) quantum computing [18] is that large Gaussian entangled (cluster) states [23] can be produced experimentally in a one-shot deterministic fashion [24,25]. Further, CV qubit states, such as the GKP qubit is known to be the most loss-resilient encoding of the qubit in a bosonic mode [26] that admit deterministic Clifford gates using Gaussian unitaries, and there are native CV quantum codes to correct for loss errors [27]. Since Gaussian states are not universal [20], one needs a non-Gaussian operation to enable universal quantum computing [28]. Experimentally, the most readily-available non-Gaussian resource is photon number resolving (PNR) detection [29]. One common 
modality in which a PNR detector can be used to probabilistically engineer non-Gaussian states is photon subtraction [30,31], which also is known to increase entanglement [32]. Photon subtraction from multimode Gaussian states has been achieved experimentally [33-35] and several theoretical aspects of photon subtraction have been studied [36-43]. In this paper, we consider a more general and simpler to describe set-up, namely partial $P N R$ detection [44,45], i.e., employing PNR detection on a subset of the modes of a multimode Gaussian state, to herald the undetected modes in a desired state, conditioned on the PNR detectors' click pattern on the detected modes. The heralded state is non-Gaussian unless all PNR detectors register zero photon clicks. This is because the projection on vacuum is a Gaussian operation and therefore will not impart any non-Gaussianity to the heralded state). We point out that partial measurements have been used before in different contexts, e.g., to realize minimal-disturbance measurements experimentally [46].

The most general multimode Gaussian state is described by a covariance matrix and a coherent displacement vector. Zeromean Gaussian states are those whose displacement vector, or the mean-field amplitude, is zero. A general $K$-mode Gaussian state can be produced by passing $K$ displaced-squeezed states through a linear-optical unitary transformation, which in turn admits a systematic design in terms of $K(K-1) 50-50$ beamsplitters and an equal number of unspecified phase elements $[13,14]$. Experimentally, the most challenging part in the above is preparing a displaced squeezed state. Preparation of squeezed vacuum state, or two-mode squeezed vacuum state of light — both of which are zero-mean Gaussian states-on the other hand, is routinely performed using spontaneous parametric downconversion, e.g., using a $\chi^{(2)}$ nonlinear medium. Recent experiments have demonstrated on-chip squeezedvacuum generation $[47,48]$. Further, since fully programmable linear optical circuits have also been realized on-chip [49], scalable generation of arbitrary multimode zero-mean Gaussian states is well within the reach of modern technology. This is the reason why we focus in this paper, on evaluating whether arbitrary non-Gaussian states can be prepared just by partial-PNR detection on zero-mean Gaussian states.

This paper is organized as follows: In Sec. II we explain how photon subtraction and addition can be seen as a special case of partial PNR. Said section can be skipped by the experienced reader but it possesses some pedagogical value, and it sets notation. In Sec. III we review briefly the mathematical description of partial PNR (see also Refs. [45,50]) and we also provide a simple proof that any pure zero-mean Gaussian state engineered with partial PNR will necessarily give a zero-mean non-Gaussian state. In Sec. IV we present the fidelity between the heralded state and a given non-Gaussian (target) state. The idea behind the upper bound on said fidelity is based on the Cauchy-Schwartz inequality. In Sec. V, we calculate said bound for any product state of single-mode superposition of the binary phase-shift keyed (BPSK) coherent states $|-\gamma\rangle,|\gamma\rangle$ and give a few examples. In Sec. VI we calculate fidelity upper bounds for multimode entangled states that are superpositions of multimode coherent states where each mode is from the BPSK constellation. The first special case of an entangled state of this type that we consider is the coherent GHZ state, for which our fidelity upper bound comes out to
1 (a trivial upper bound). However, for the coherent-cat basis cluster states (CCCS), our fidelity upper bound evaluates to $1 / 2$, showing such a state cannot be prepared by partial PNR on a zero-mean Gaussian state. Finally, in Sec. VII, we summarize our results: We discuss how the fidelity upper bound relates to the absence of a nonzero mean (or coherent displacement), we put our findings in context with the literature on non-Gaussian quantum state preparation, present further intuition, and briefly discuss future directions of research.

\section{PARTIAL PNR AS A GENERALIZATION OF PHOTON SUBTRACTION AND ADDITION}

Let us consider photon subtraction on the most general single-mode Gaussian pure state: a squeezed-coherent state $\left|\alpha_{1}, r_{1}\right\rangle$, where $\alpha_{1}, r_{1} \in \mathbb{C}$ are the displacement and squeezing parameters, respectively. The state interacts with vacuum on a beam-splitter of transmissivity $\tau$. On the reflective (lowtransmissivity) output port of the beam-splitter, PNR detection is performed, which registers, say, $n_{1}$ photons. This heralds the subtraction of $n_{1}$ photons from the input state. This conditional photon-subtracted state $\left|\Phi_{n_{1}}\right\rangle$-i.e., the state heralded on the transmitted port of the beam-splitter-is non-Gaussian whenever $n_{1} \geqslant 1$. One can write down the probability of detecting $n_{1}$ photons (and hence producing $\left|\Phi_{n_{1}}\right\rangle$ ) as a function of $\alpha_{1}, r_{1}$, and $\tau$ [45]. A natural generalization of this setup is to allow for further Gaussian resources by substituting the vacuum state at the other input of the beam-splitter with another single mode Gaussian pure state $\left|\alpha_{2}, r_{2}\right\rangle, \alpha_{2}, r_{2} \in \mathbb{C}$, and then proceed with PNR detection on one output port, and considering the heralded state on the other output port if $n_{1}$ photons are detected. No matter what figure of merit we might choose on the quality of the heralded state (e.g., fidelity to some target state), it can only improve or, at worst, remain the same as compared to that using photon subtraction. This is because vacuum is a trivial special case of a pure Gaussian state. We term this setup partial PNR detection [44]: PNR detection on one mode of a two-mode general Gaussian pure state to seek a desired postselected non-Gaussian state on the undetected mode.

Next, let us consider photon subtraction on all modes of a $K$-mode pure Gaussian state (which in general is entangled), by coupling the $i$ th mode with vacuum on a beam-splitter of transmissivity $\tau_{i}, i=1, \ldots, K$. If we count all the ancillary vacuum states as input modes, we have a $2 K$-mode Gaussian state $K$ modes of which are detected with PNR, resulting in a $K$-mode (generally non-Gaussian) state. An obvious generalization is to consider an $N$-mode Gaussian pure state (where $N$ can be even or odd) and to apply PNR detection on $N-M$ modes, resulting in an $M$-mode state $\left|\Phi_{n_{M+1}, \ldots, n_{N}}\right\rangle$, conditioned on the PNR pattern $\left(n_{M+1} \ldots, n_{N}\right)$.

Let us note that partial PNR detection schemes incorporates multiple photon addition as well. Photon addition is modeled utilizing a beam-splitter whose upper input is the state $|\Psi\rangle$ (or a mode of the state) which will undergo photon addition while in the beam-splitter's lower input port a Fock state $|n\rangle$ is injected. A PNR detector is applied on the lower output port which if heralds $m \leqslant n$ photons, then photons have been added to the state $|\Psi\rangle$, resulting to a state $|\Phi\rangle$ which in general will be non-Gaussian. To produce a Fock state $|n\rangle$, 


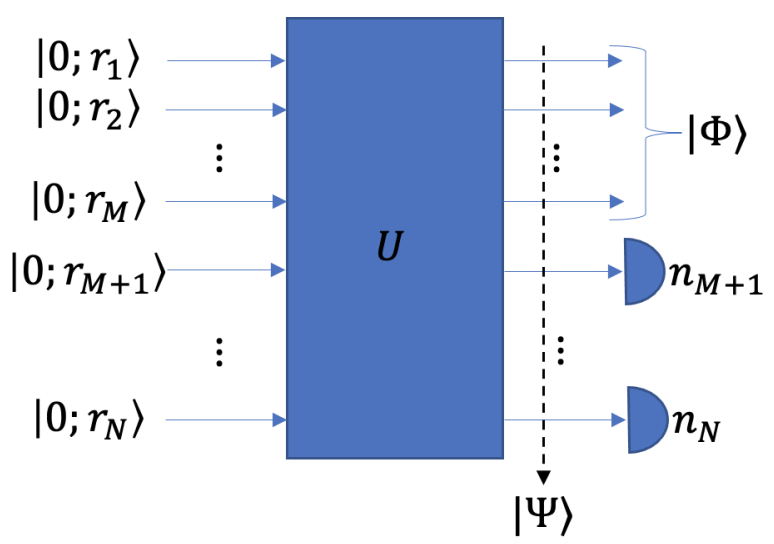

FIG. 1. Generic scheme of heralding an $M$-mode state $|\Phi\rangle$ from an $N$-mode zero-mean Gaussian state $|\Psi\rangle$. The state $|\Psi\rangle$ is produced by the interaction of $N$ squeezed vacuum states through a general passive (beam-splitters and phase shifters) Gaussian unitary operation $U$. Partial PNR detection on the $N-M$ lower modes of $|\Psi\rangle$ produces a non-Gaussian state $|\Phi\rangle$ on the undetected $M$ modes.

i.e., the input to the lower port of the beam-splitter, one can consider a two-mode squeezed vacuum state (TMSV) whose one mode is detected using a PNR detector. Then, this Fock state can be used for the photon addition task. Equivalently, one can include several TMSV states as part of a general Gaussian state and all the PNR detectors to be included in the very last step of the state engineering protocol.

\section{MATHEMATICAL DESCRIPTION OF PARTIAL PNR ON ZERO-MEAN GAUSSIAN STATES}

Let us consider a zero-mean $N$-mode Gaussian (in general entangled) pure state $|\Psi\rangle$, prepared by mixing $N$ single-mode squeezed vacuum states of squeezing parameters $r_{i}, 1 \leqslant i \leqslant$ $N$, in a linear optical $N$-mode unitary operation $U$. We consider partial PNR detection on $N-M$ of those modes and the generic setup is shown in Fig. 1. In Ref. [45] and Ref. [50] it was shown that conditioned on the PNR detection pattern $\left(n_{M+1} \ldots, n_{N}\right)$, the $M$-mode heralded state $\left|\Phi_{n_{M+1}, \ldots, n_{N}}\right\rangle \equiv$ $|\Phi\rangle$ can be written in the Fock basis as,

$$
|\Phi\rangle=\sum_{n_{1}, \ldots, n_{M}=0}^{\infty} c_{n_{1} \ldots n_{M}}\left|n_{1} \ldots n_{M}\right\rangle,
$$

where

$$
c_{n_{1} \ldots n_{M}}=\frac{\mathcal{I}_{n_{1} \ldots n_{M} n_{M+1} \ldots n_{N}}}{\sqrt{P} \prod_{i=1}^{N} \sqrt{n_{i} ! 2^{n_{i}} \cosh r_{i}}} .
$$

The probability of obtaining the PNR pattern $\left(n_{M+1}, \ldots, n_{N}\right)$ is given by

$$
P=\sum_{n_{1}, \ldots, n_{M}=0}^{\infty} \frac{\left|\mathcal{I}_{n_{1} \ldots n_{N}}\right|^{2}}{\prod_{i=1}^{N} n_{i} ! 2^{n_{i}} \cosh r_{i}}
$$

and

$$
\mathcal{I}_{n_{1} \ldots n_{N}}=\left\{\begin{array}{ll}
0 & \sum_{i=1}^{N} n_{i}=\text { odd } \\
\operatorname{haf}(\sigma) & \sum_{i=1}^{N} n_{i}=\text { even }
\end{array},\right.
$$

where the hafnian [51] in Eq. (4) is evaluated for a matrix $\sigma$ whose elements are defined as

$$
\sigma_{i j}=2\left(\mathcal{H}_{i j}^{-1}-\mathcal{H}_{i+N}^{-1}{ }_{j+N}\right),
$$

where

$$
\mathcal{H}^{-1}=\frac{1}{2}\left[\begin{array}{cc}
3 I-A-i C & i(A-I+i C) \\
i(A-I+i C) & I+A+i C
\end{array}\right],
$$

and $A$ and $C$ are block matrices that define the covariance matrix,

$$
\Gamma^{-1}=\left(\begin{array}{cc}
A & C \\
C & 2 I-A
\end{array}\right)
$$

of the $Q$ function of $|\Psi\rangle$, i.e., of the form $Q(\vec{R})=$ $\left[(2 \pi)^{N} \sqrt{\operatorname{det} \Gamma}\right]^{-1} \exp \left[-\vec{R}^{T} \Gamma^{-1} \vec{R} / 2\right], \quad$ where $\quad \vec{R}^{T}=$ $\left(q_{1}, \ldots, p_{N}\right)$ are the phase-space coordinates.

We note that the formulas of this section can be found in Ref. [45] and Ref. [50]. The details of state $|\Psi\rangle$ (i.e., its covariance matrix) are not relevant to this work and we just provide Eqs. (5), (6), and (7) for completeness. The rest of the expressions of this section can be found in Ref. [45].

Without loss of generality, we set the modes undergoing PNR to be the "last" $N-M$ modes of $|\Psi\rangle$. Further, we consider $r_{i}>0$ to be real valued, or equivalently, all phases are pushed into the passive interferometer $U$ that entangles the squeezed vacuum states to create the resource Gaussian state $|\Psi\rangle$.

For the main result of this paper, we will not need to invoke the explicit dependence of $\sigma$ on $|\Psi\rangle$, through the squeezing parameters $r_{i}$ and the parameters of the entangling passive linear-optical unitary $U$. The property of importance to us will be the parity of the PNR detector's pattern in Eq. (4).

Let us now prove that if the $N$-mode Gaussian resource state $|\Psi\rangle$ is zero mean, then the heralded $M$-mode conditional state $|\Phi\rangle$ is also zero mean. In other words, the mean-field amplitude of $|\Phi\rangle$ is zero, i.e., $\left\langle\Phi\left|\hat{a}_{i}\right| \Phi\right\rangle \equiv\left\langle\hat{a}_{i}\right\rangle_{\Phi}=0$ for all $i \in\{1, \ldots, M\}$. Expressing this condition in the Fock basis, we have:

$$
\left\langle\hat{a}_{i}\right\rangle_{\Phi}=\sum_{n_{1}, \ldots, n_{M}=0}^{\infty} \sqrt{n_{i}+1} c_{n_{1} \ldots n_{i} \ldots n_{M}} c_{n_{1} \ldots n_{i}+1 \ldots n_{M}}^{*} .
$$

As per Eqs. (2) and (4), the coefficients $c_{n_{1} \ldots n_{i} \ldots n_{M}}$ (and hence their complex conjugates) are nonzero only if $n_{1}+\cdots+n_{i}$ $+\cdots+n_{N}=$ even. Therefore, for each nonzero term in the sum above, $n_{1}+\cdots+n_{i}+\cdots+n_{N}=$ even, and hence $n_{1}+\cdots+n_{i}+1+\cdots+n_{N}=$ odd. Hence, $c_{n_{1} \ldots n_{i}+1 \ldots n_{M}}^{*}=$ 0 , rendering every term in the sum to be zero. Therefore, $\left\langle\hat{a}_{i}\right\rangle_{\Phi}=0$.

\section{FIDELITY UPPER BOUND ON THE CONDITIONAL STATE}

In Sec. III we proved that a zero-mean Gaussian pure state under partial PNR will necessarily give a zero-mean conditional state on the unmeasured modes. Therefore, it is natural to anticipate that any non-Gaussian target state with an arbitrary nonzero mean-field would not have a fidelity arbitrarily close to 1 with a non-Gaussian state engineered using partial PNR on zero-mean Gaussian states. However, 
the question of what the highest said fidelity can be, remains open. In this section, we provide a general recipe to find an upper bound to the fidelity for any target state. In subsequent sections, we will apply this technique to evaluate our fidelity upper bound on specific non-Gaussian states of interest.

The fidelity $\mathcal{F}=\left|\left\langle\Phi_{t} \mid \Phi\right\rangle\right|^{2}$ between the conditional state $|\Phi\rangle$ of Eq. (1) and a non-Gaussian target state $\left|\Phi_{t}\right\rangle=$ $\sum_{n_{1}, \ldots, n_{M}=0}^{\infty} d_{n_{1} \ldots n_{M}}\left|n_{1} \ldots n_{M}\right\rangle$ reads

$$
\mathcal{F}=\left|\sum_{n_{1}, \ldots, n_{M}=0}^{\infty} c_{n_{1} \ldots n_{M}}^{*} d_{n_{1} \ldots n_{M}}\right|^{2}
$$

It is apparent that if we use the Cauchy-Schwartz inequality on Eq. (8), we will get $\mathcal{F} \leqslant 1$. However, we will see that under the constraint [Eqs. (2) and (4)] $n_{1}+\cdots+n_{N}=$ even, the Cauchy-Schwartz inequality gives a nontrivial upper bound. Then $n_{1}+\cdots+n_{M}=$ even if the summation of the PNR pattern $\left(n_{M+1}, \ldots, n_{N}\right)$ is even, and $n_{1}+\cdots+n_{M}=$ odd if the summation of the PNR pattern $\left(n_{M+1}, \ldots, n_{N}\right)$ is odd. Therefore, we rewrite Eq. (8) as

$$
\mathcal{F}=\left\{\begin{array}{ll}
\mathcal{F}_{\text {even }}, & \sum_{i=n_{M+1}}^{N} n_{i}=\text { even } \\
\mathcal{F}_{\text {odd }}, & \sum_{i=n_{M+1}}^{N} n_{i}=\text { odd }
\end{array},\right.
$$

where

$$
\begin{aligned}
& \mathcal{F}_{\text {even }}=\left|\sum_{\substack{n_{1}, \ldots, n_{M}=0 \\
n_{1}+\ldots+n_{M}=\text { even }}}^{\infty} c_{n_{1} \ldots n_{M}}^{*} d_{n_{1} \ldots n_{M}}\right|^{2}, \\
& \mathcal{F}_{\text {odd }}=\left|\sum_{\substack{n_{1}, \ldots, n_{M}=0 \\
n_{1}+\cdots+n_{M}=\text { odd }}}^{\infty} c_{n_{1} \ldots n_{M}}^{*} d_{n_{1} \ldots n_{M}}\right|^{2} .
\end{aligned}
$$

Let us consider the case where $n_{1}+\cdots+n_{M}=$ even. Then $\mathcal{F}_{\text {odd }}=0$ and we can use the Cauchy-Schwartz inequality to get

$$
\mathcal{F}_{\text {even }} \leqslant \sum_{\substack{n_{1}, \ldots, n_{M}=0 \\ n_{1}+\cdots+n_{M}=\text { even }}}^{\infty}\left|c_{n_{1} \ldots n_{M}}\right|^{2} \sum_{\substack{n_{1}, \ldots, n_{M}=0 \\ n_{1}+\cdots+n_{M}=\text { even }}}^{\infty}\left|d_{n_{1} \ldots n_{M}}\right|^{2} .
$$

Finally, exploiting the fact that the state $|\Phi\rangle$ has nonzero coefficients under the constraint $n_{1}+\cdots+n_{M}=$ even, we write

$$
\sum_{\substack{n_{1}, \ldots, n_{M}=0 \\ n_{1}+\cdots+n_{M}=\text { even }}}^{\infty}\left|c_{n_{1} \ldots n_{M}}\right|^{2}=1
$$

and we get

$$
\mathcal{F}_{\text {even }} \leqslant \sum_{\substack{n_{1}, \ldots, n_{M}=0 \\ n_{1}+\cdots+n_{M}=\text { even }}}^{\infty}\left|d_{n_{1} \ldots n_{M}}\right|^{2}=u_{\text {even }} .
$$

Similarly, for the complementary case where $n_{1}+\cdots+n_{M}=$ odd, we have that $\mathcal{F}_{\text {even }}=0$ and

$$
\mathcal{F}_{\text {odd }} \leqslant \sum_{\substack{n_{1}, \ldots, n_{M}=0 \\ n_{1}+\cdots+n_{M}=\text { odd }}}^{\infty}\left|d_{n_{1} \ldots n_{M}}\right|^{2}=u_{\text {odd }}
$$

Four observations are necessary here. First, we observe that $0 \leqslant u_{\text {even }} \leqslant 1$ and $0 \leqslant u_{\text {odd }} \leqslant 1$ and both bounds depend only on the target state, and therefore they are easy to compute. Second, the non-Gaussian target state is normalized, and therefore

$$
u_{\text {odd }}=1-u_{\text {even }} \text {. }
$$

It is possible that for the desired non-Gaussian target state, $u_{\text {even }}$ and $u_{\text {odd }}$ to be unequal. In that case, we will use as upper bound the larger among the two and herald on the PNR pattern whose parity corresponds to that of the higher upper bound. Third, let us note that we view fidelity as necessary criterion for successful non-Gaussian state engineering. For example, a coherent cat state $N_{0}^{-1}(|\gamma\rangle+|-\gamma\rangle$ ) (where $| \pm \gamma\rangle$ is a coherent state and $N_{0}$ is normalization) can have high fidelity with vacuum for small, albeit nonzero, $\gamma$ amplitude. However, vacuum and small coherent cat states are inherently different. On the other hand, if one derives a low-enough upper bound for the fidelity, then the impossibility of producing the state under consideration is certain. Last, we observe that assuming a zero-mean Gaussian resource state, resulted to imposing a specific parity on the PNR pattern. The question now is how this parity constraint impacts the state engineering performance.

\section{FIDELITY UPPER BOUNDS FOR COHERENT CAT PRODUCT STATES}

Consider a single mode state $|c\rangle$, which is a superposition of two coherent states $| \pm \gamma\rangle$,

$$
|c\rangle=b_{1}|\gamma\rangle+b_{2}|-\gamma\rangle,
$$

where $b_{1}, b_{2} \in \mathbb{C}$ satisfy

$$
\left(b_{1} b_{2}^{*}+b_{1}^{*} b_{2}\right) e^{-2|\gamma|^{2}}=1-\left|b_{1}\right|^{2}-\left|b_{2}\right|^{2},
$$

so that $\langle c \mid c\rangle=1$. Let us calculate the upper bounds of Eqs. (13) and (14) for the product state $|c\rangle^{\otimes M}$. Following Eq. (13), we have

$$
u_{\text {even }}=\sum_{\substack{n_{1}, \ldots, n_{M}=0 \\ n_{1}+\cdots+n_{M}=\text { even }}}^{\infty}\left|\left\langle n_{1} \mid c\right\rangle\right|^{2} \ldots\left|\left\langle n_{M} \mid c\right\rangle\right|^{2},
$$

which can be rewritten as

$$
u_{\text {even }}=\sum_{n_{1}, \ldots, n_{M}=0}^{\infty}\left|\left\langle n_{1} \mid c\right\rangle\right|^{2} \ldots\left|\left\langle n_{M} \mid c\right\rangle\right|^{2} \frac{1+(-1)^{n_{1}+\cdots+n_{M}}}{2} .
$$

By separating the fraction of Eq. (18) and using the fact that state $|c\rangle^{\otimes M}$ is normalized we get

$$
u_{\text {even }}=\frac{1}{2}+\frac{1}{2} \sum_{n_{1}, \ldots, n_{M}=0}^{\infty}\left|\left\langle n_{1} \mid c\right\rangle\right|^{2} \ldots\left|\left\langle n_{M} \mid c\right\rangle\right|^{2}(-1)^{n_{1}+\cdots+n_{M}} .
$$

Using Eq. (16), the Fock basis expansion of a coherent state $|\gamma\rangle=\exp \left(-|\gamma|^{2} / 2\right) \sum_{n=0}^{\infty} \gamma^{n} / \sqrt{n !}|n\rangle$, and Eq. (17), we find

$$
u_{\text {even }}=\frac{1}{2}+\frac{1}{2}\left[e^{2|\gamma|^{2}}-2\left(\left|b_{1}\right|^{2}+\left|b_{2}\right|^{2}\right) \sinh \left(2|\gamma|^{2}\right)\right]^{M},
$$


and using Eq. (15) we get

$$
u_{\text {odd }}=\frac{1}{2}-\frac{1}{2}\left[e^{2|\gamma|^{2}}-2\left(\left|b_{1}\right|^{2}+\left|b_{2}\right|^{2}\right) \sinh \left(2|\gamma|^{2}\right)\right]^{M} .
$$

We observe that $u_{\text {even }}$ and $u_{\text {odd }}$ for the state of Eq. (16) depend only on the absolute values of the the state's coefficients when expressed as a coherent states' superposition. We note that for our $N$-mode Gaussian state, the $M$-mode produced state, and the $M$ target states, we allow $N$ and $M, 1 \leqslant M<N$, to be arbitrary.

As applications, we will consider the following target states:

$$
\begin{gathered}
|\overline{0}\rangle=\frac{1}{N_{0}}(|\gamma\rangle+|-\gamma\rangle), \\
|\overline{1}\rangle=\frac{1}{N_{1}}(|\gamma\rangle-|-\gamma\rangle), \\
|+\rangle=\frac{1}{\sqrt{2}}(|\overline{0}\rangle+|\overline{1}\rangle), \\
|-\rangle=\frac{1}{\sqrt{2}}(|\overline{0}\rangle-|\overline{1}\rangle),
\end{gathered}
$$

where $N_{k}=\sqrt{2\left[1+(-1)^{k} e^{-2|\gamma|^{2}}\right]}, \quad k=0,1$. The states of Eqs. (22), (23), (24), and (25), are the computationaland rotated-basis qubit states corresponding to the coherent cat-basis qubit - one of the leading qubit candidates for allphotonic quantum computing. The states $|\overline{0}\rangle$ and $|\overline{1}\rangle$ form the so-called logical qubit basis, while the states $|+\rangle$ and $|-\rangle$ are derived by the action of a Hadamard gate (defined on the qubit basis) on the logical qubit basis' kets. For the $|\overline{0}\rangle,|\overline{1}\rangle$ states we find

$$
\begin{gathered}
u_{\mathrm{even}}^{|\overline{0}\rangle^{\otimes M}}=1, \\
u_{\mathrm{odd}}^{|\overline{0}\rangle^{\otimes M}}=0, \\
u_{\mathrm{even}}^{|\overline{1}\rangle^{\otimes M}}=\frac{1+(-1)^{M}}{2}, \\
u_{\mathrm{odd}}^{|\overline{1}\rangle^{\otimes M}}=\frac{1-(-1)^{M}}{2} .
\end{gathered}
$$

Consistent with the parity of the $|\overline{0}\rangle,|\overline{1}\rangle$ states, we see that a PNR pattern whose summation is odd cannot herald the state $|\overline{0}\rangle$, while the state $|\overline{0}\rangle$ is not impossible to be engineered if the summation of the PNR pattern is an even number. Also, if $M$ is an odd (even) number, then $|\overline{1}\rangle$ cannot be heralded if the PNR pattern is summed to an even (odd) number. We note that an upper bound equal to 1 does not mean that the state can be engineered with perfect fidelity. However, high fidelity for generating approximations of the $|\overline{0}\rangle,|\overline{1}\rangle$ states has been found in the literature $[45,50,52]$ using the partial PNR method, even with the resource Gaussian state being zero mean.

For the $|+\rangle,|-\rangle$ states we find

$$
\begin{aligned}
& u_{\text {even }}^{|+\rangle^{\otimes M}}=\frac{1}{2}, \\
& u_{\text {odd }}^{|+\rangle^{\otimes M}}=\frac{1}{2}, \\
& u_{\text {even }}^{|-\rangle^{\otimes M}}=\frac{1}{2}, \\
& u_{\text {odd }}^{|-\rangle^{\otimes M}}=\frac{1}{2} .
\end{aligned}
$$

Since $|+\rangle,|-\rangle$ are not zero-mean states, we expect that the upper bound should reflect that by being less than 1 . In fact, the upper bounds are low enough to conclude that the $|+\rangle,|-\rangle$ states cannot be heralded no matter what the summation of the PNR pattern is. Let us assume that we can engineer the $|\overline{0}\rangle$ or $|\overline{1}\rangle$ state with perfect fidelity from a zero-mean Gaussian state using partial PNR. Then, since it is impossible to engineer the $|+\rangle,|-\rangle$ states utilizing a zero-mean Gaussian state, we conclude that any optical implementation of a Hadamard gate (defined in the qubit space) based on Gaussian resources and partial PNR, must necessarily include displacements, in accordance with the setups presented in [53].

\section{FIDELITY UPPER BOUNDS FOR COHERENT GHZ AND CLUSTER STATES}

Consider a non-Gaussian target state that is the multimode superposition,

$$
|C\rangle=\sum_{l=1}^{2^{M}} b_{l}\left|\boldsymbol{\gamma}^{(l)}\right\rangle,
$$

where $b_{l} \in \mathbb{C}$ are such that state $|C\rangle$ is normalized and $\left|\boldsymbol{\gamma}^{(l)}\right\rangle$ is a product of $M$ coherent states $|\gamma\rangle,|-\gamma\rangle$, or any combination thereof (there exist $2^{M}$ such product states). We can rewrite Eq. (34) as

$$
|C\rangle=\sum_{l=1}^{2^{M}} b_{l}(-1)^{\boldsymbol{v}(l) \cdot \hat{\boldsymbol{n}}}|\boldsymbol{\gamma}\rangle,
$$

where $|\boldsymbol{\gamma}\rangle \equiv\left|\boldsymbol{\gamma}^{(1)}\right\rangle$ is a product of $M$ coherent states $|\gamma\rangle, \boldsymbol{v}(l)$ is a vector consisting of $M$ components which are combinations of \pm 1 [e.g., $\boldsymbol{v}(1)=(1, \ldots, 1), \boldsymbol{v}(2)=(-1,1, \ldots)$, $\boldsymbol{v}(3)=(1,-1,1, \ldots, 1)$, there exist $2^{M}$ such vectors $]$, and $\hat{\boldsymbol{n}}=\left(\hat{n}_{1}, \ldots, \hat{n}_{M}\right)$. Equation (13) gives

$$
\begin{aligned}
u_{\text {even }} & =\sum_{\substack{n_{1}, \ldots, n_{M}=0 \\
n_{1}+\cdots+n_{M}=\text { even }}}^{\infty}\left|\left\langle n_{1} \ldots n_{M} \mid C\right\rangle\right|^{2} \\
& =\sum_{n_{1}, \ldots, n_{M}=0}^{\infty}\left|\left\langle n_{1} \ldots n_{M} \mid C\right\rangle\right|^{2}\left[\frac{1+(-1)^{n_{1}+\cdots+n_{M}}}{2}\right] .
\end{aligned}
$$

Working out Eq. (36) and using (35) we get

$$
\begin{aligned}
u_{\text {even }}= & \frac{1}{2}+\frac{e^{-M|\gamma|^{2}}}{2} \sum_{n_{1}, \ldots, n_{M}=0}^{\infty} \frac{|\gamma|^{2\left(n_{1}+\cdots+n_{M}\right)}}{n_{1} ! \ldots n_{M} !} \\
& \times\left|\sum_{l=1}^{2^{M}} b_{l}(-1)^{\boldsymbol{v}(l) \cdot \boldsymbol{n}}\right|^{2}(-1)^{n_{1}+\cdots+n_{M}},
\end{aligned}
$$

where $\boldsymbol{n}=\left(n_{1}, \ldots, n_{M}\right)$. It is hard to write Eq. (37) in closed form; however, if one specifies the coefficients $b_{l}$, the summation is rendered computable. One could write an expression that is similar to Eq. (37) for a state like (34) but with different coherent amplitudes per mode; however, states with equal coherent amplitudes are relevant to quantum computing. We remind the reader that the upper bound $u_{\text {odd }}$ is always given by $u_{\text {odd }}=1-u_{\text {even }}$ as per Eq. (15). 


\section{A. GHZ states}

Let us examine the following GHZ states:

$$
\left|\mathrm{GHZ}_{ \pm}\right\rangle=\frac{1}{N_{ \pm}}(|\boldsymbol{\gamma}\rangle \pm|-\boldsymbol{\gamma}\rangle),
$$

where $N_{ \pm}=\sqrt{2\left(1 \pm e^{-2 M|\gamma|^{2}}\right)}$ are the normalization constants. Applying Eq. (37) and (15) we find

$$
\begin{aligned}
& u_{\text {even }}^{\left|\mathrm{GHZ}_{+}\right\rangle}=1, \\
& u_{\text {odd }}^{\left|\mathrm{GHZ}_{+}\right\rangle}=0, \\
& u_{\text {even }}^{\left|\mathrm{GHZ}_{-}\right\rangle}=0, \\
& u_{\text {odd }}^{\left|\mathrm{GHZ}_{-}\right\rangle}=1,
\end{aligned}
$$

which is again consistent with the fact that the mean filed amplitude of GHZ states is zero and with the parity of the PNR pattern imposed by the absence of displacement in the resource Gaussian state. We note again that we do not prove that our upper bound is attainable. However, it has been shown that GHZ states can be produced with high fidelity [45] even with zero-mean resource Gaussian states.

\section{B. Coherent cat-basis cluster state}

Let us move to a more interesting case. Consider the $C Z_{2}$ gate whose action is defined as $C Z_{2}|\overline{0} \overline{0}\rangle=|\overline{0} \overline{0}\rangle, C Z_{2}|\overline{0} \overline{1}\rangle=$ $|\overline{0} \overline{1}\rangle, C Z_{2}|\overline{1} \overline{0}\rangle=|\overline{1} \overline{0}\rangle$, and $C Z_{2}|\overline{1} \overline{1}\rangle=-|\overline{1} \overline{1}\rangle$ and therefore is an entangling operation when it acts on $|++\rangle$. In this work, we denote as $C Z$ any product consisting of multiple two-mode $C Z_{2}$ gates, acting on any two qubits of a multiqubit product state. In fact, we consider that $C Z$ acts on the state $|+\rangle^{\otimes M}$, i.e., $C Z|+\rangle^{\otimes M}$, to create entanglement between any possible couple of $|+\rangle$ states at the same time, therefore creating a cluster state on the coherent-cat basis, i.e.,

$$
|\mathrm{CCCS}\rangle=C Z|+\rangle^{\otimes M}
$$

is any coherent cat-basis cluster state. Using Eq. (24), said state can be written as

$$
\begin{aligned}
|\mathrm{CCCS}\rangle= & \frac{1}{2^{M / 2}}\left[s_{1}|\overline{0} \ldots \overline{0}\rangle+s_{2}|\overline{1} \overline{0} \ldots \overline{0}\rangle+\ldots\right. \\
& +s_{M+2}|\overline{0} \ldots \overline{0} \overline{1}\rangle+s_{M+3}|\overline{1} \overline{1} \ldots \overline{0}\rangle \\
& +\cdots+s_{\left(\begin{array}{c}
M \\
2
\end{array}\right)+(M+3)}|\overline{0} \ldots \overline{1} \overline{1}\rangle \\
& \left.+\cdots+s_{2^{M}}|\overline{1} \ldots \overline{1}\rangle\right] .
\end{aligned}
$$

Let us explain the terms of Eq. (44): The first line is the product state $|\overline{0}\rangle^{\otimes M}$ and there is $\left(\begin{array}{c}M \\ 0\end{array}\right)=1$ such state. The second line is a product of $M-1|\overline{0}\rangle$ states and $1|\overline{1}\rangle$ state which can take any position and therefore there are $\left(\begin{array}{c}M \\ 1\end{array}\right)=M$ such states present in said line. Similarly, in the third line the $2|\overline{1}\rangle$ states can take any position and there are $\left(\begin{array}{c}M \\ 2\end{array}\right)$ such states, and so on. Finally, in the last line all states are $|\overline{1}\rangle$ and there is only one such state as $\left(\begin{array}{l}M \\ M\end{array}\right)=1$. The prefactors $s_{i}, i=1, \ldots, 2^{M}$ can only be \pm 1 , according to the prescription of any given $C Z$ gate creating any desired cluster configuration. In fact, unless there are at least two $|\overline{1}\rangle$ vectors present, one can set $s_{i}=1, \forall i$.
From Eqs. (13) and (44) we have

$$
\begin{aligned}
u_{\text {even }}^{|\mathrm{CCCS}\rangle}= & \frac{1}{2^{M}} \sum_{\substack{n_{1}, \ldots, n_{M}=0 \\
n_{1}+\cdots+n_{M}=\text { even }}}^{\infty} \mid s_{1}\left\langle n_{1} \ldots n_{M} \mid \overline{0} \ldots \overline{0}\right\rangle+\ldots \\
& +\left.s_{2^{M}}\left\langle n_{1} \ldots n_{M} \mid \overline{1} \ldots \overline{1}\right\rangle\right|^{2} .
\end{aligned}
$$

Let us examine the cross-terms of the expansion of $|\ldots|^{2}$ in Eq. (45), i.e., terms with different prefactors $s_{i}$. Any such term is the product $\left\langle n_{1} \ldots n_{M} \mid \bar{q}_{1} \ldots \bar{q}_{M}\right\rangle\left\langle\bar{q}_{1}^{\prime} \ldots \bar{q}_{M}^{\prime} \mid n_{1} \ldots n_{M}\right\rangle$, with $\bar{q}_{1}, \ldots, \bar{q}_{M}=0,1$ and $\bar{q}_{1}^{\prime}, \ldots, \bar{q}_{M}^{\prime}=0,1$. Since they are cross-terms, there is at least one $i=1, \ldots, M$ such that $\bar{q}_{i} \neq \bar{q}_{i}^{\prime}$. Given the Fock expansion coefficients $\quad\langle n \mid \overline{0}\rangle=N_{0}^{-1} e^{-|\gamma|^{2} / 2} \gamma^{n}\left[1+(-1)^{n}\right] / \sqrt{n !} \quad$ and $\langle n \mid \overline{1}\rangle=N_{1}^{-1} e^{-|\gamma|^{2} / 2} \gamma^{n}\left[1-(-1)^{n}\right] / \sqrt{n !}$, we see that any cross-term will be proportional to $\left[1+(-1)^{n_{i}}\right]\left[1-(-1)^{n_{i}}\right]=$ $1-(-1)^{2 n_{i}}=0$, for at least one $i$.

Therefore, the only nonzero terms in Eq. (45) are of the form $\left|s_{i}\left\langle n_{1} \ldots n_{M} \mid \bar{q}_{1} \ldots \bar{q}_{M}\right\rangle\right|^{2}=\left|\left\langle n_{1} \ldots n_{M} \mid \bar{q}_{1} \ldots \bar{q}_{M}\right\rangle\right|^{2}$, since $\left|s_{i}\right|^{2}=1$. Therefore, we have

$$
u_{\mathrm{even}}^{|\mathrm{CCCS}\rangle}=\frac{1}{2^{M}} \sum_{k=0}^{M}\left(\begin{array}{c}
M \\
k
\end{array}\right) u_{\text {even }}^{\left.||^{\otimes(M-k)} \overline{1}^{\otimes k}\right\rangle} .
$$

From Eq. (13) and following the methods of Sec. V, we find that

$$
u_{\text {even }}^{\left|\overline{0}^{\otimes(M-k)} \overline{1}^{\otimes k}\right\rangle}=\frac{1+(-1)^{k}}{2} .
$$

Finally, from Eqs. (46), (47), and (15) we find

$$
\begin{aligned}
& u_{\text {even }}^{|\mathrm{CCCS}\rangle}=\frac{1}{2}, \\
& u_{\text {odd }}^{|\mathrm{CCCS}\rangle}=\frac{1}{2} .
\end{aligned}
$$

Any $|\mathrm{CCCS}\rangle$ has inherently nonzero mean-field amplitude because the $|+\rangle$ has nonzero displacement. The upper bound of Eqs. (48) and (49) quantifies the damage of not considering displacement as a resource. An upper bound equal to $1 / 2$ on the fidelity with any produced state shows that any $|\mathrm{CCCS}\rangle$ state is well beyond reach with a zero-mean Gaussian resource state.

\section{CONCLUSIONS AND DISCUSSION}

Partial PNR is the new trend for non-Gaussian bosonic state engineering because essentially it circumvents the technical difficulties of constructing non-Gaussian optical unitary operations. However, the are two main drawbacks in said approach: (i) optimization methods are needed to reveal an optimal resource Gaussian state that maximizes the fidelity and probability of occurrence of an acceptable produced state (ii) fidelity is merely a necessary criterion. Any numerical optimization typically does not give intuition on the underlying physics of state engineering. In this paper we asked what would happen if we forbid our resource state to possess any displacements and therefore reveal the implications on coherent-cat basis clusters under any optimization of such resource. We recognized that zero displacement restricts the parity of the observed PNR pattern and therefore it restricts the Fock expansion coefficients (modulo squared) one should 
sum up to derive a fidelity upper bound, yielding a hard $1 / 2$ upper bound for target states with nonzero mean-field amplitude such as the $|+\rangle,|-\rangle$, and $|\mathrm{CCCS}\rangle$ states.

As a by-product, we argued that any optical implementation, i.e., based on Gaussian resources and partial PNR, of a qubit Hadamard gate (an operation transforming $|\overline{0}\rangle \rightarrow|+\rangle$ ) must necessarily include displacements. This Hadamard gate could be a separate module consisting $m$ displaced squeezed states as inputs to a passive $n$-mode interferometer $(m<n)$. The rest of the $n-m$ input modes could be the output state of another partial PNR-based scheme which produces the $|\overline{0}\rangle$ or $|\overline{1}\rangle$ states. The Hadamard optical module and the $|\overline{0}\rangle$ or $|\overline{1}\rangle$ state generator could be combined into a single interferometer, with single mode dispalced squeezed inputs, and an array of PNR detectors at the output, some of which control the production of $|\overline{0}\rangle$ or $|\overline{1}\rangle$ and another PNR subset the realization of the Hadamard gate.

It is known that PNR detectors and Gaussian states comprise a universal resource set [28]. Therefore, by working with a general pure Gaussian state, i.e., including displacements, universality must be restored. Apparently, a displacement $D(\alpha)$ on the undetected output would not suffice as it can be easily seen that for example $D(\alpha)|\overline{0}\rangle \neq|+\rangle$. All displacements must be applied on the squeezed single mode states going into the passive interferometer or just before partial PNR (i.e., equivalently partially projecting a zero-mean Gaussian state onto displaced Fock states). However, a constructive way of designing partial PNR-based schemes which would attain universality is still elusive.
The holy grail of this line of research would be a systematic theory for non-Gaussian state engineering for specific classes of states that are useful in various quantum information processing tasks such as cluster states for quantum computing, all-optical quantum repeaters, metrologically optimal states in distributed quantum sensing, etc. One specific interesting question that relates to the states considered in this paper is as follows: Whether the GHZ states considered in this work can be transformed into the CCCS by using local unitaries (e.g., it is known that a star-topology cluster state and a GHZ state are local-Hadamard equivalent [54]), where the local unitaries are themselves realized by postselected non-Gaussian ancilla states which in turn were heralded using Gaussian states and PNR detectors [53].

Such questions could be answered by expanding the mathematical formalism developed in Refs. [45,50] to include displacements. This could catalyze further progress toward the non-Gaussian state engineering, if not in providing constructive ways for attaining universality, but at least for constructing optical implementations for specific useful to quantum computation modules.

\section{ACKNOWLEDGMENTS}

C.N.G. was supported by Xanadu Quantum Technologies. C.N.G. and S.G. acknowledge Xanadu Quantum Technologies and Linran Fan for multiple useful discussions on this topic. C.N.G. also acknowledges partial funding support from the National Science Foundation, FET, Award No. 2122337.
[1] M. Pant, D. Towsley, D. Englund, and S. Guha, Percolation thresholds for photonic quantum computing, Nat. Commun. 10, 1070 (2019).

[2] S. Guha, Structured Optical Receivers to Attain Superadditive Capacity and the Holevo Limit, Phys. Rev. Lett. 106, 240502 (2011).

[3] M. Sabuncu, R. Filip, G. Leuchs, and U. L. Andersen, Environment-assisted quantum-information correction for continuous variables, Phys. Rev. A 81, 012325 (2010).

[4] K. Azuma, K. Tamaki, and H.-K. Lo, All-photonic quantum repeaters, Nat. Commun. 6, 6787 (2015).

[5] M. Pant, H. Krovi, D. Englund, and S. Guha, Rate-distance tradeoff and resource costs for all-optical quantum repeaters, Phys. Rev. A 95, 012304 (2017).

[6] M. He, R. Malaney, and J. Green, Global entanglement distribution with multi-mode non-gaussian operations, IEEE J. Sel. Areas Commun. 38, 528 (2020).

[7] S.-H. Tan, B. I. Erkmen, V. Giovannetti, S. Guha, S. Lloyd, L. Maccone, S. Pirandola, and J. H. Shapiro, Quantum Illumination with Gaussian States, Phys. Rev. Lett. 101, 253601 (2008).

[8] S. Guha and B. I. Erkmen, Gaussian-state quantum-illumination receivers for target detection, Phys. Rev. A 80, 052310 (2009).

[9] Q. Zhuang, Z. Zhang, and J. H. Shapiro, Optimum Mixed-State Discrimination for Noisy Entanglement-Dnhanced Sensing, Phys. Rev. Lett. 118, 040801 (2017).

[10] J. P. Dowling, Quantum optical metrology-the lowdown on high-n00n states, Contemp. Phys. 49, 125 (2008).
[11] P. C. Humphreys, M. Barbieri, A. Datta, and I. A. Walmsley, Quantum Enhanced Multiple Phase Estimation, Phys. Rev. Lett. 111, 070403 (2013).

[12] S. Pirandola, B. R. Bardhan, T. Gehring, C. Weedbrook, and S. Lloyd, Advances in photonic quantum sensing, Nat. Photon. 12, 724 (2018)

[13] M. Reck, A. Zeilinger, H. J. Bernstein, and P. Bertani, Experimental Realization of Any Discrete Unitary Operator, Phys. Rev. Lett. 73, 58 (1994).

[14] W. R. Clements, P. C. Humphreys, B. J. Metcalf, W. S. Kolthammer, and I. A. Walmsley, Optimal design for universal multiport interferometers, Optica 3, 1460 (2016).

[15] S. L. Braunstein, Squeezing as an irreducible resource, Phys. Rev. A 71, 055801 (2005).

[16] E. Knill, R. Laflamme, and G. J. Milburn, A scheme for efficient quantum computation with linear optics, Nature (Lond.) 409, 46 (2001).

[17] P. Kok, W. J. Munro, K. Nemoto, T. C. Ralph, J. P. Dowling, and G. J. Milburn, Linear optical quantum computing with photonic qubits, Rev. Mod. Phys. 79, 135 (2007).

[18] D. Gottesman, A. Kitaev, and J. Preskill, Encoding a qubit in an oscillator, Phys. Rev. A 64, 012310 (2001).

[19] J. H. Shapiro, Single-photon kerr nonlinearities do not help quantum computation, Phys. Rev. A 73, 062305 (2006).

[20] J. Niset, J. Fiurášek, and N. J. Cerf, No-Go Theorem for Gaussian Quantum Error Correction, Phys. Rev. Lett. 102, 120501 (2009). 
[21] M. Gimeno-Segovia, P. Shadbolt, D. E. Browne, and T. Rudolph, From Three-Photon Greenberger-Horne-Zeilinger States to Ballistic Universal Quantum Computation, Phys. Rev. Lett. 115, 020502 (2015).

[22] A. Olivo and F. Grosshans, Ancilla-assisted linear optical bell measurements and their optimality, Phys. Rev. A 98, 042323 (2018).

[23] C. González-Arciniegas, P. Nussenzveig, M. Martinelli, and O. Pfister, Cluster states from gaussian states: Essential diagnostic tools for continuous-variable one-way quantum computing, PRX Quant. 2, 030343 (2021).

[24] M. Pysher, Y. Miwa, R. Shahrokhshahi, R. Bloomer, and O. Pfister, Parallel Generation of Quadripartite Cluster Entanglement in the Optical Frequency Comb, Phys. Rev. Lett. 107, 030505 (2011).

[25] S. Yokoyama, R. Ukai, S. C. Armstrong, C. Sornphiphatphong, T. Kaji, S. Suzuki, J.-i. Yoshikawa, H. Yonezawa, N. C. Menicucci, and A. Furusawa, Ultra-large-scale continuousvariable cluster states multiplexed in the time domain, Nat. Photon. 7, 982 (2013).

[26] K. Noh, V. V. Albert, and L. Jiang, Quantum capacity bounds of gaussian thermal loss channels and achievable rates with Gottesman-Kitaev-Preskill codes, IEEE Trans. Inf. Theory 65 , 2563 (2019).

[27] M. Lassen, M. Sabuncu, A. Huck, J. Niset, G. Leuchs, N. J. Cerf, and U. L. Andersen, Quantum optical coherence can survive photon losses using a continuous-variable quantum erasure-correcting code, Nat. Photon. 4, 700 (2010).

[28] S. Lloyd and S. L. Braunstein, Quantum Computation over Continuous Variables, Phys. Rev. Lett. 82, 1784 (1999).

[29] B. Cabrera, R. M. Clarke, P. Colling, A. J. Miller, S. Nam, and R. W. Romani, Detection of single infrared, optical, and ultraviolet photons using superconducting transition edge sensors, Appl. Phys. Lett. 73, 735 (1998).

[30] J. Fiurášek, R. García-Patrón, and N. J. Cerf, Conditional generation of arbitrary single-mode quantum states of light by repeated photon subtractions, Phys. Rev. A 72, 033822 (2005).

[31] A. Ourjoumtsev, F. Ferreyrol, R. Tualle-Brouri, and P. Grangier, Preparation of non-local superpositions of quasi-classical light states, Nat. Phys. 5, 189 (2009).

[32] A. Ourjoumtsev, A. Dantan, R. Tualle-Brouri, and P. Grangier, Increasing Entanglement between Gaussian States by Coherent Photon Subtraction, Phys. Rev. Lett. 98, 030502 (2007).

[33] Y.-S. Ra, C. Jacquard, A. Dufour, C. Fabre, and N. Treps, Tomography of a Mode-Tunable Coherent Single-Photon Subtractor, Phys. Rev. X 7, 031012 (2017).

[34] Y.-S. Ra, A. Dufour, M. Walschaers, C. Jacquard, T. Michel, C. Fabre, and N. Treps, Non-gaussian quantum states of a multimode light field, Nat. Phys. 16, 144 (2020).

[35] H. Takahashi, K. Wakui, S. Suzuki, M. Takeoka, K. Hayasaka, A. Furusawa, and M. Sasaki, Generation of Large-Amplitude Coherent-State Superposition via Ancilla-Assisted Photon Subtraction, Phys. Rev. Lett. 101, 233605 (2008).

[36] V. Averchenko, C. Jacquard, V. Thiel, C. Fabre, and N. Treps, Multimode theory of single-photon subtraction, New J. Phys. 18, 083042 (2016).

[37] R. Tualle-Brouri, A. Ourjoumtsev, A. Dantan, P. Grangier, M. Wubs, and A. S. Sørensen, Multimode model for projective photon-counting measurements, Phys. Rev. A 80, 013806 (2009).
[38] P. Marek, H. Jeong, and M. S. Kim, Generating "squeezed" superpositions of coherent states using photon addition and subtraction, Phys. Rev. A 78, 063811 (2008).

[39] S. M. Barnett, G. Ferenczi, C. R. Gilson, and F. C. Speirits, Statistics of photon-subtracted and photon-added states, Phys. Rev. A 98, 013809 (2018).

[40] M. Walschaers, S. Sarkar, V. Parigi, and N. Treps, Tailoring Non-Gaussian Continuous-Variable Graph States, Phys. Rev. Lett. 121, 220501 (2018).

[41] F. Arzani, A. Ferraro, and V. Parigi, High-dimensional quantum encoding via photon-subtracted squeezed states, Phys. Rev. A 99, 022342 (2019).

[42] M. Walschaers, C. Fabre, V. Parigi, and N. Treps, Statistical signatures of multimode single-photon-added and -subtracted states of light, Phys. Rev. A 96, 053835 (2017).

[43] U. Chabaud, T. Douce, D. Markham, P. van Loock, E. Kashefi, and G. Ferrini, Continuous-variable sampling from photonadded or photon-subtracted squeezed states, Phys. Rev. A 96, 062307 (2017).

[44] D. Su, C. R. Myers, and K. K. Sabapathy, Conversion of Gaussian states to non-gaussian states using photon-numberresolving detectors, Phys. Rev. A 100, 052301 (2019).

[45] C. N. Gagatsos and S. Guha, Efficient representation of gaussian states for multimode non-gaussian quantum state engineering via subtraction of arbitrary number of photons, Phys. Rev. A 99, 053816 (2019).

[46] M. Sabuncu, L. Mišta, J. Fiurášek, R. Filip, G. Leuchs, and U. L. Andersen, Nonunity gain minimal-disturbance measurement, Phys. Rev. A 76, 032309 (2007).

[47] Y. Zhang, M. Menotti, K. Tan, V. D. Vaidya, D. H. Mahler, L. G. Helt, L. Zatti, M. Liscidini, B. Morrison, and Z. Vernon, Squeezed light from a nanophotonic molecule, Nat. Commun. 12, 2233 (2021).

[48] Z. Yang, M. Jahanbozorgi, D. Jeong, S. Sun, O. Pfister, H. Lee, and $\mathrm{X}$. Yi, A squeezed quantum microcomb on a chip, Nat. Commun. 12, 4781 (2021).

[49] N. C. Harris, J. Carolan, D. Bunandar, M. Prabhu, M. Hochberg, T. Baehr-Jones, M. L. Fanto, A. Matthew Smith, C. C. Tison, P. M. Alsing, and D. Englund, Linear programmable nanophotonic processors, Optica 5, 1623 (2018).

[50] A. J. Pizzimenti, J. M. Lukens, H.-H. Lu, N. A. Peters, S. Guha, and C. N. Gagatsos, Non-gaussian photonic state engineering with the quantum frequency processor, arXiv:2108.08290 [quant-ph] [Phys. Rev. A (to be published)].

[51] Actually this will give a loop-hanfian $[44,50]$ since more than one photons can be detected in any one of the PNR detectors. A loop-hafnian can be treated as a usual hafnian or it can be more efficiently calculated by using the formula found in Ref. [[55], Prop. 1, p. 547]. We note, however, that in this work we do not engage in calculating the hafnian.

[52] M. Dakna, T. Anhut, T. Opatrný, L. Knöll, and D.-G. Welsch, Generating Schrödinger-cat-like states by means of conditional measurements on a beam-splitter, Phys. Rev. A 55, 3184 (1997).

[53] T. C. Ralph, A. Gilchrist, G. J. Milburn, W. J. Munro, and S. Glancy, Quantum computation with optical coherent states, Phys. Rev. A 68, 042319 (2003).

[54] S. Aaronson and D. Gottesman, Improved simulation of stabilizer circuits, Phys. Rev. A 70, 052328 (2004).

[55] R. Kan, From moments of sum to moments of product, J. Multivar. Anal. 99, 542 (2008). 